\title{
Ginsenoside-Mc1 reduces ischemia/ reperfusion-induced cardiac arrhythmias through activating JAK2/STAT3 pathway and attenuating oxidative/endoplasmic reticulum stress in hyperlipidemic rats
}

https://doi.org/10.1515/tjb-2021-0171

Received August 6, 2021; accepted November 15, 2021; published online February 21, 2022

\begin{abstract}
Objectives: Patients suffering from myocardial ischemiareperfusion (IR) injuries usually have varying degrees of negatively-affecting comorbidities like hyperlipidemia. We evaluated the preconditioning effect of ginsenoside-Mc1 on reperfusion injury-induced myocardial arrhythmias, along with cardiac oxidative stress, endoplasmic reticulum stress protein expression, and histological damage in hyperlipidemic rats, and further, explore the role of JAK2/STAT3 activity.

Methods: Thirty-five Sprague-Dawley rats fed a high-fat diet for eight weeks. Ginsenoside-Mc1 (10 mg/dL, IP) was administered to hyperlipidemic rats daily for one month before IR injury. IR injury was induced by 35 min LAD coronary artery ligation and subsequent 60 -min reperfusion. A selective JAK2 inhibitor (AG490) was injected before IR injury. Electrocardiography was recorded and myocardial arrhythmias (ventricular premature complexes, tachycardia, and fibrillation) were evaluated in the reperfusion phase according to Lambeth convention. Hematoxylin-Eosin staining, spectrophotometry, and Western blotting techniques were employed to measure the endpoints.
\end{abstract}

\footnotetext{
*Corresponding author: Yanwei Zhang, Teaching and Research Section of Internal Medicine, The First Clinical Medical College of Shanxi Medical University, No. 85 Xinjian South Road, Taiyuan City, Shanxi Province, 030001, P.R. China, E-mail: zyw001c@sina.com. https://orcid.org/0000-0002-9552-2850

Kun Xu and Yanqing Zhang, Teaching and Research Section of Internal Medicine, The First Clinical Medical College of Shanxi Medical University, Taiyuan City, Shanxi Province, P.R. China. https:// orcid.org/0000-0002-7123-5195 (K. Xu). https://orcid.org/00000001-9202-4365 (Y. Zhang)
}

Results: IR injury in hyperlipidemic rats significantly increased the reperfusion-induced myocardial arrhythmias counts, timing, incidence, and severity. The expression of proteins of endoplasmic reticulum stress (p-PERK, p-eIF2 $\alpha, \mathrm{CHOP}$ ), and oxidative stress marker malondialdehyde were significantly upregulated following IR induction, whereas antioxidant superoxide-dismutase and JAK2/STAT3 proteins expression significantly reduced, as compared with untreated-hyperlipidemic rats. Administration of ginsenoside-Mc1 to hyperlipidemic rats significantly corrected the arrhythmogenic feature of IR injury, reduced phosphorylation and expression of PERK, eIF2 $\alpha$, CHOP, and improved oxidative stress and histological changes. Interestingly, inhibition of the JAK2/STAT3 pathway via AG490 significantly abolished ginsenosideMc1-induced cardioprotection.

Conclusions: Taken together, ginsenoside-Mc1 exerts substantial anti-arrhythmogenic influences against myocardial IR injury in hyperlipidemic rats through activation of JAK2/STAT3 pathway and subsequent reduction of oxidative/endoplasmic reticulum stress.

Keywords: cardioprotection; comorbidity; endoplasmic reticulum; ginsenoside; hyperlipidemia; oxidative stress.

\section{Introduction}

Despite recent advances in medicine and surgical interventions, ischemic heart disease is still the leading cause of death in the world [1]. Most patients suffering from myocardial ischemia-reperfusion (IR) injuries have varying degrees of other comorbidities such as obesity, hyperlipidemia, and diabetes. Hyperlipidemia and hypercholesterolemia are one of the main risk factors for myocardial diseases associated with IR injury and can exacerbate IR-induced heart damages [2]. Reperfusion-induced 
myocardial arrhythmias are of determining factors in the survival or death of patients with myocardial infarction (MI) whose severity and incidence increase in the presence of hyperlipidemia [2-4]. In animal models of hyperlipidemia, endogenous cardiac defense mechanisms are attenuated and lose their normal function, leading to the development of oxidative stress, apoptosis, and cell death [5]. Based on plenty of evidence, the Janus kinase-2/signal transducer and activator of transcription-3 (JAK2/STAT3) pathway has also an important contribution in cardioprotection against myocardial IR injury [6, 7]. JAK2/STAT3 has antioxidative features and belongs to the survivor activating factor enhancement (SAFE) signaling pathway that its activity is considerably reduced in the setting of hyperlipidemia $[5,8]$. These findings highlight the significance of activation of this pathway in improving the clinical outcomes of MI patients.

The detrimental effects of hyperlipidemia in cardioprotection can also be due to an overproduction of reactive oxygen species (ROS) such as superoxide free radicals and hydrogen peroxide $[8,9]$. The exacerbation of oxidative stress caused by these reactions triggers apoptosis and negatively affects cell membranes, proteins, and cardiomyocytes' organelles. The endoplasmic reticulum (ER) plays an important role in controlling numerous intracellular physiological functions (including calcium homeostasis, lipid biosynthesis, protein folding) [10]. Pathophysiological conditions of IR injury and hyperlipidemia disrupt ER homeostasis and activate the unfolded protein response (UPR) and ER stress, leading to the occurrence of arrhythmias [10, 11]. Prolonged ER stress in hyperlipidemic conditions induces oxidative stress, inflammation, and tissue apoptosis by activating ER transmembrane sensors and UPR initiation [12]. PERK and eIF2 $\alpha$ proteins are among these ER stress sensors. With the onset of oxidative stress, the PERK signaling pathway inhibits protein synthesis by reducing the protein load in the ER lumen and induces phosphorylation of eIF2 $\alpha$ [10]. Subsequently, these processes lead to cell death by activating the transcription factor ATE 4 and CHOP. A few documents reported that JAK2/STAT3 signaling may prevent ER stress during myocardial IR injury [7, 13], leading to the reduction of cardiac arrhythmias incidence. However, the exact role of ER stress is not yet well understood not only in the pathophysiology of IR-induced cardiac arrhythmias but also in the effect of hyperlipidemia on the severity of IR injury.

Ginsenoside-compound Mc1 (GMC) is a de-glycosylated ginsenoside extracted from ginseng and has important effects in reducing oxidative stress, inflammatory and apoptotic reactions $[14,15]$. A study has shown that this compound decreases oxidative stress in H9c2 cells and exerts promising effects in reducing the myocardial infarct size in obese mice [14]. It has recently been reported that ginsenoside compounds can lower hyperlipidemia in mice model [15]. GMC also improved liver steatosis and insulin resistance by attenuating ER stress [16]. Considering these beneficial potentials of GMC in reducing oxidative/ER stress, it seems to have a significant impact on reducing arrhythmias and blunting arrhythmogenic mechanisms of reperfusion injury in hyperlipidemic conditions. It would also be interesting to explore whether GMC cardioprotective action is associated with JAK2/STAT3 signaling. Therefore, in this study, we aimed to test this hypothesis and evaluate the effect of pretreatment of hyperlipidemic rats with GMC on the incidence and severity of ventricular arrhythmias as well as the expression of proteins involved in ER stress, oxidative stress, and histological damage in an in-vivo model of myocardial IR injury and further explore the role of JAK2/STAT3 activity in these effects.

\section{Materials and methods}

\section{Animals}

Male Sprague-Dawley rats with an initial weight of $250 \pm 20$ gr were purchased from the Animal Center of the University and transferred to the animal room under standard conditions. Mice were kept in ethylene cages in $12 \mathrm{~h}$ of light/dark cycle at $24^{\circ} \mathrm{C}$ and $55 \%$ humidity. All animal experiments and interventions in this study were performed in accordance with international ethical standards based on the approval of the University Ethics Committee (Ethics number: SDFRE2019023Dx).

\section{Induction of hyperlipidemia and myocardial ischemia- reperfusion injury}

To induce hyperlipidemia, all rats were fed a high-fat diet regimen (containing 45\% fat, 20\% protein, and 35\% carbohydrate). The duration of receiving a high-fat diet was two months [5, 17]. After two months of receiving a hyperlipidemic regimen, rats were anesthetized with sodium pentobarbital ( $50 \mathrm{mg} / \mathrm{kg}$, i.p) and heparinized (500 unit). Following thoracotomy by cutting the 4th and 5th intercostal muscles, the hearts were exposed and the left anterior descending (LAD) coronary artery was ligated with a 4-0 silk thread for $35 \mathrm{~min}$ to induce regional ischemia. Then, the thread was removed and reperfusion was applied for $60 \mathrm{~min}$ by reopening the LAD coronary artery [2].

\section{Animal grouping}

The animals were randomly divided into the following 5 groups with 7 rats in each.

(1) Hyperlipidemic (HL) group, in which rats received a high-fat diet for two months and then subjected to thoracotomy without IR injury. 
(2) Hyperlipidemic + myocardial IR ( $\mathrm{HL}+\mathrm{MI})$ group, in which HL rats were subjected to IR injury.

(3) Hyperlipidemic + myocardial IR + ginsenoside (HL + MI + GMC) group, in which $\mathrm{HL}$ rats received GMC $(10 \mathrm{mg} / \mathrm{dL}, \mathrm{IP})$ [14] daily for one month in the second month of the high-fat regimen period, and then subjected to IR injury.

(4) Hyperlipidemic + myocardial IR + JAK2 inhibitor (HL + MI + AG490) group, in which $\mathrm{HL}$ rats received AG $4905 \mathrm{mg} / \mathrm{kg}$, IP, daily for 7 days before IR injury with one-day intervals, and $20 \mathrm{mg} / \mathrm{kg}$, IP, $10 \mathrm{~min}$ after the onset of thoracotomy [18], and then subjected to IR injury.

(5) Hyperlipidemic + myocardial IR + ginsenoside + JAK2 inhibitor $(\mathrm{HL}+\mathrm{MI}+\mathrm{GMC}+\mathrm{AG} 490)$ group, in which HL rats received both GMC and AG490 at their corresponding time points, and then subjected to IR injury.

GMC and AG490 were prepared in DMSO plus normal saline. Rats in groups 1 and 2 were also injected intraperitoneally with the vehicle at intervals of 2 days, for one month. Using a sample size calculation software, sample size analysis revealed a required number of rats being 6 for each group. However, we considered 7 rats per group to increase the analysis power in the study.

\section{Recording and interpretation of myocardial arrhythmias}

Electrocardiographs were recorded by connecting three electrodes to both right and left hands and right foot on the axis lead II during the IR experiments. Ventricular arrhythmias including premature ventricular complexes (PVC), ventricular tachycardia (VT), and ventricular fibrillation (VF) were evaluated during the reperfusion phase based on the Lambeth convention (Figure 1). The count and duration of PVC, VT, and VF, the incidence of VT and VF as well as the severity of arrhythmias were calculated. The following scoring system was employed to calculate the severity of arrhythmias: 0: no arrhythmia; 1: only PVC; 2: PVC plus 1-3 episode of VT; 3: PVC plus

Normal ECG
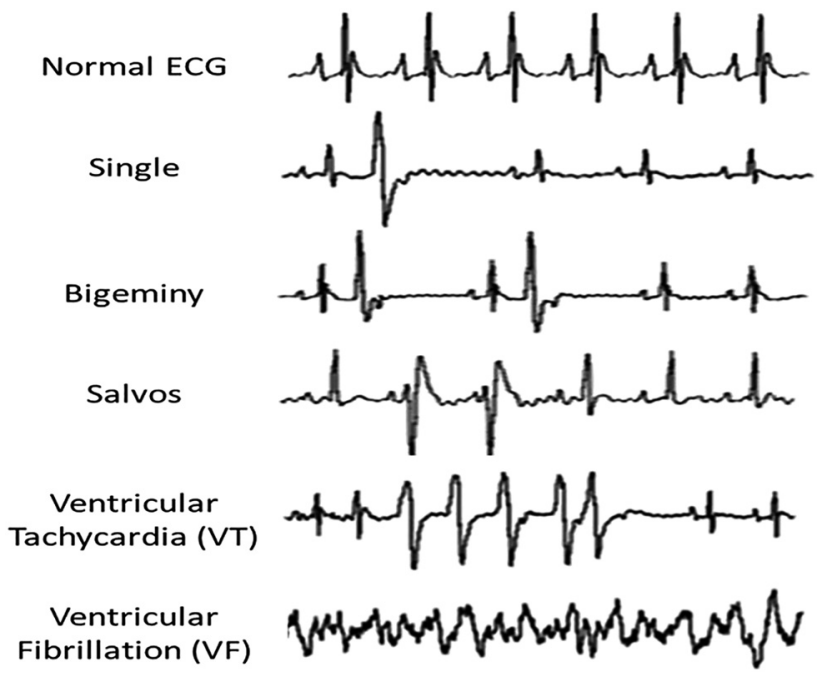

Figure 1: Types of myocardial arrhythmias according to the Lambeth convention. PVC, premature ventricular complexes; VT, ventricular tachycardia; and VF, ventricular fibrillation. Single beats, bigeminy and salvos were considered as PVCs. more episodes of VT; 4. PVC plus VT and reversible VF; 5 PVC plus VT and irreversible VF.

\section{Histological assessments}

Hematoxylin-Eosin (H\&E) staining was used for histological examination. At the end of reperfusion, $250 \mathrm{mg}$ of the ischemic zone of the left ventricle was removed and immersed in 10\% formalin. Then 4$5 \mu \mathrm{m}$ sections were obtained and successive steps of tissue washing, passage, and dehydrating processes were performed and the samples were stained with H\&E. Histological changes such as intercellular spaces expanding and edema, infiltration of inflammatory cells, and necrosis were assessed by a blind histologist using a light microscope at five visual fields.

\section{Measurement of lipid profile}

Before the induction of hyperlipidemia and after 8 weeks, lipid profiles were measured in blood samples obtained from tail vein of rats. The concentrations of cholesterol, triglyceride, and low- and high-density lipoproteins (LDL and HDL) were measured with commercially available kits (Wako Pure Chemical Industries, Osaka, Japan), according to the manufacturer's instructions.

\section{Measurement of oxidative stress indices}

After sampling $(100 \mathrm{mg})$ from the left ventricular ischemic zone and freezing them with liquid nitrogen, the samples were homogenized on ice and then centrifuged at $13,000 \mathrm{rpm}$ for $10 \mathrm{~min}$ at $4^{\circ} \mathrm{C}$ to measure the levels of lipid peroxidation marker (malondialdehyde, MDA) and the antioxidant enzyme superoxide dismutase (SOD). MDA was measured by thiobarbituric acid reaction and SOD enzyme by enzymatic spectrophotometric method, according to the instructions of the manufacturer's kits. The values were normalized and reported according to the protein amount of each sample. The sample protein content was estimated by the Bradford method.

\section{Western blotting}

The western blotting technique was used to evaluate the expression and phosphorylation of proteins involved in ER stress including PERK, eIF-2a, and CHOP. Briefly, after electrophoresis of approximately $10 \mathrm{mg}$ of protein derived from each sample, the proteins were transferred to a PVDF membrane. Then, the PVDF membrane was incubated with $5 \%$ skim milk for $1 \mathrm{~h}$ at laboratory temperature. After washing with PBS three times, proteins were incubated with primary antibodies against p-PERK, p-eIF2 $\alpha$, CHOP, and GAPDH (1:1500, Cell Signaling Technology, USA). After $24 \mathrm{~h}$, the membranes were washed again and exposed to HRP-conjugated secondary antibodies for $1 \mathrm{~h}$. The membrane was then washed again and incubated with enhanced chemiluminescence reagents and the resulting bands were visualized using a visualizer machine. Band intensities in different samples were calculated by Image J software and normalized with GAPDH band intensities (as internal control). 


\section{Statistical analysis}

The data were expressed as Mean \pm SD Parametric variables changes between different groups were analyzed by one-way ANOVA, followed by Tukey post hoc test. A p-value less than 0.05 was considered statistically significant.

\section{Results}

\section{General characteristics of the rats}

After feeding the high-fat diet to rats for 8 weeks, the hyperlipidemic or HL rats had significantly higher body weights and heart weights than their initial (baseline) values $(p<0.01)$. In addition, the plasma levels of cholesterol, LDL $(\mathrm{p}<0.01)$ and triglyceride $(\mathrm{p}<0.05)$ were significantly higher, and the plasma level of HDL ( $\mathrm{p}<0.05)$ was lower in HL rats after 8 weeks in comparison to the corresponding baseline values. Administration of GMC to $\mathrm{HL}$ rats significantly reduced the body weights, and the levels of cholesterol and LDL $(\mathrm{p}<0.05)$, without affecting on hearts weights, heart/ body weights ratio, and the levels of triglyceride and HDL, as compared with the final values of untreated HL rats (Table 1 ).

\section{GMC reduced IR-induced ventricular arrhythmias, whereas these effects were reversed by AG490 co-treatment}

Induction of MI by IR injury in hyperlipidemic rats led to a significant increase in the number (Figure 2A) and duration (Figure 2B) of PVC, VT, and VF arrhythmias in reperfusion phase as compared with untreated hyperlipidemic rats

Table 1: General information of hyperlipidemic rats before and after feeding high-fat diet.

\begin{tabular}{lrrr}
\hline Parameters & $\begin{array}{r}\text { Baseline } \\
\text { values in HL } \\
\text { rats }(\mathbf{n}=\mathbf{2 1})\end{array}$ & $\begin{array}{r}\text { Final values in } \\
\text { untreated HL } \\
\text { rats }(\mathbf{n}=14)\end{array}$ & $\begin{array}{r}\text { Final values in } \\
\text { GMC-treated } \\
\text { HL rats }(\mathbf{n}=7)\end{array}$ \\
\hline $\mathrm{BW}, \mathrm{g}$ & $253 \pm 12$ & $417 \pm 14^{\mathrm{b}}$ & $370 \pm 12^{\mathrm{bc}}$ \\
$\mathrm{HW}, \mathrm{g}$ & $1.24 \pm 0.06$ & $1.73 \pm 0.10^{\mathrm{a}}$ & $1.56 \pm 0.11$ \\
$\mathrm{HW} / \mathrm{BW}, \%$ & $0.49 \pm 0.01$ & $0.42 \pm 0.02$ & $0.43 \pm 0.01$ \\
Cholesterol, mg/dL & $81 \pm 9$ & $186 \pm 21^{\mathrm{b}}$ & $144 \pm 17^{\mathrm{bc}}$ \\
Triglyceride, $\mathrm{mg} / \mathrm{dL}$ & $107 \pm 11$ & $146 \pm 19$ & $132 \pm 13$ \\
LDL-C, $\mathrm{mg} / \mathrm{dL}$ & $41 \pm 2$ & $80 \pm 7^{\mathrm{b}}$ & $63 \pm 5^{\mathrm{bc}}$ \\
$\mathrm{HDL}-\mathrm{C}, \mathrm{mg} / \mathrm{dL}$ & $55 \pm 4$ & $38 \pm 3^{\mathrm{a}}$ & $41 \pm 3^{\mathrm{a}}$ \\
\hline
\end{tabular}

The data were expressed as mean $\pm S D,{ }^{a} p<0.05$ and ${ }^{b} p<0.01$ vs. baseline values; and ${ }^{c} p<0.05$ vs. final values in untreated $H L$ rats. $\mathrm{HL}$, hyperlipidemic; GMC, ginsenoside-Mc1; BW, body weight; HW, heart weight; LDL-C, low density lipoprotein cholesterol; HDL-C, high density lipoprotein cholesterol.
(HL group) ( $p<0.05$ to $p<0.001)$. GMC administration for one month significantly reduced the number of PVC and VF $(\mathrm{p}<0.05)$ and the duration of PVC, VF $(\mathrm{p}<0.001)$, and VT $(p<0.05)$ when compared to the HL + MI group. Inhibition of JAK2 by AG490, although did not have a significant effect on reperfusion-induced myocardial arrhythmias alone, inhibited the protective effect of GMC on the number and duration of all three types of arrhythmias compared with the $\mathrm{HL}+\mathrm{MI}+\mathrm{GMC}$ group (Figure 2).

The incidence of VT and VF arrhythmias following IR induction in hyperlipidemic rats increased significantly (Figure 3A). However, pretreatment of hyperlipidemic rats with GMC was able to significantly reduce the incidence of $\mathrm{VF}(\mathrm{p}<0.001)$, although its effect on reducing the incidence of VT was not statistically significant. Also, co-administration of AG490 abolished the effectiveness of GMC in reducing the incidence of these reperfusion-induced myocardial arrhythmias, especially VF ( $<<0.001)$, in comparison to the $\mathrm{HL}+\mathrm{MI}+\mathrm{GM}$ group (Figure 3A).

Finally, the severity of arrhythmias in different groups is seen in Figure 3B. GMC administration significantly reduced the reperfusion-induced increased severity of arrhythmias in hyperlipidemic rats $(\mathrm{p}<0.01)$, and this effect was partially abolished by inhibition of JAK2 by AG490 co-administration $(\mathrm{p}<0.05)$ (Figure 3B).

\section{GMC reduced myocardial IR-induced histological changes, whereas these effects were reversed by AG490 co-treatment}

The results of the histological examination showed that although lesions such as intercellular edema and inflammatory cell infiltration were observed in the HL group, induction of IR injury exacerbated these lesions and caused necrotic damages in cardiomyocytes (Figure 4). On the other hand, GMC administration significantly diminished the severity of these lesions, and co-treatment with AG490 negatively affected GMC's protective effect on histological changes.

\section{GMC reduced IR-induced myocardial oxidative stress, whereas these effects were reversed by AG490 co-treatment}

To assess the status of oxidative stress, intracellular levels of MDA as an indicator of lipid peroxidation and SOD as an endogenous antioxidant enzyme were measured. The results showed that following the induction of IR injury in 

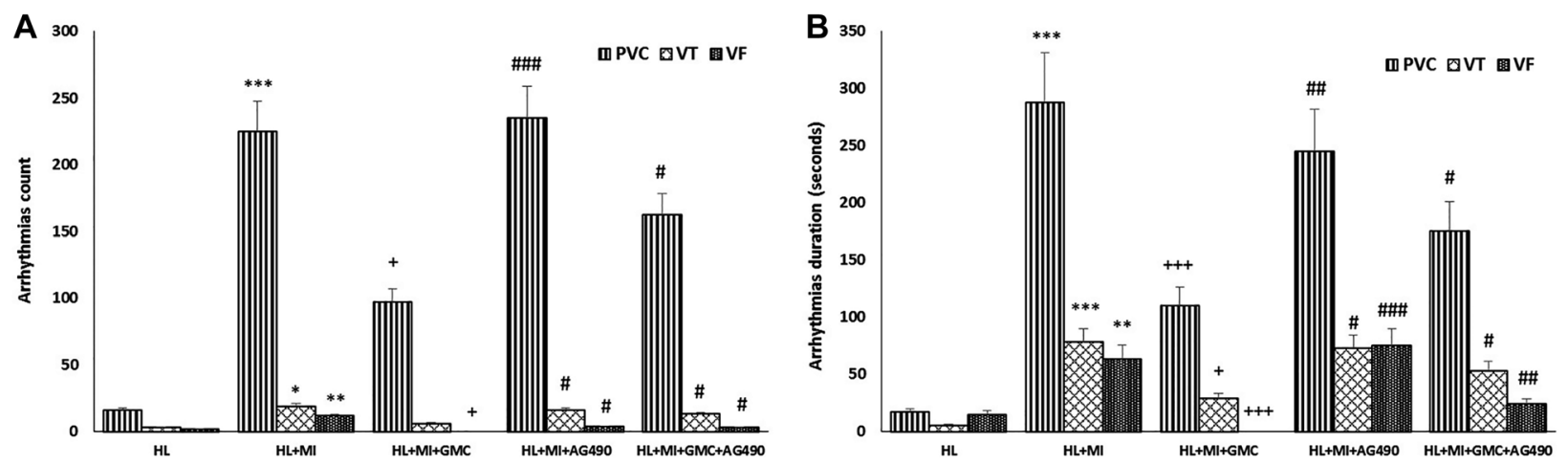

Figure 2: The effects of GMC administration on the number (A) and duration (B) of reperfusion-induced myocardial arrhythmias and their reversal by $A G 490$ in hyperlipidemic $(\mathrm{HL})$ rats.

Mean \pm SD ( $n=7$ in each group). MI, myocardial ischemia reperfusion injury; GMC, Ginsenoside-Mc1; AG490, JAK2 inhibitor; PVC, premature ventricular complexes; VT, ventricular tachycardia; and VF, ventricular fibrillation. ${ }^{\star} p<0.05,{ }^{\star \star} p<0.01,{ }^{\star \star \star} p<0.001 \mathrm{vs}$. HL group; ${ }^{+} p<0.05$, ${ }^{+++} \mathrm{p}<0.001$ vs. HL + MI group; ${ }^{\mathrm{p}}<0.05,{ }^{\#} \mathrm{p}<0.01,{ }^{\# \# \#} \mathrm{p}<0.001$ vs. $\mathrm{HL}+\mathrm{MI}+\mathrm{GMC}$ group.
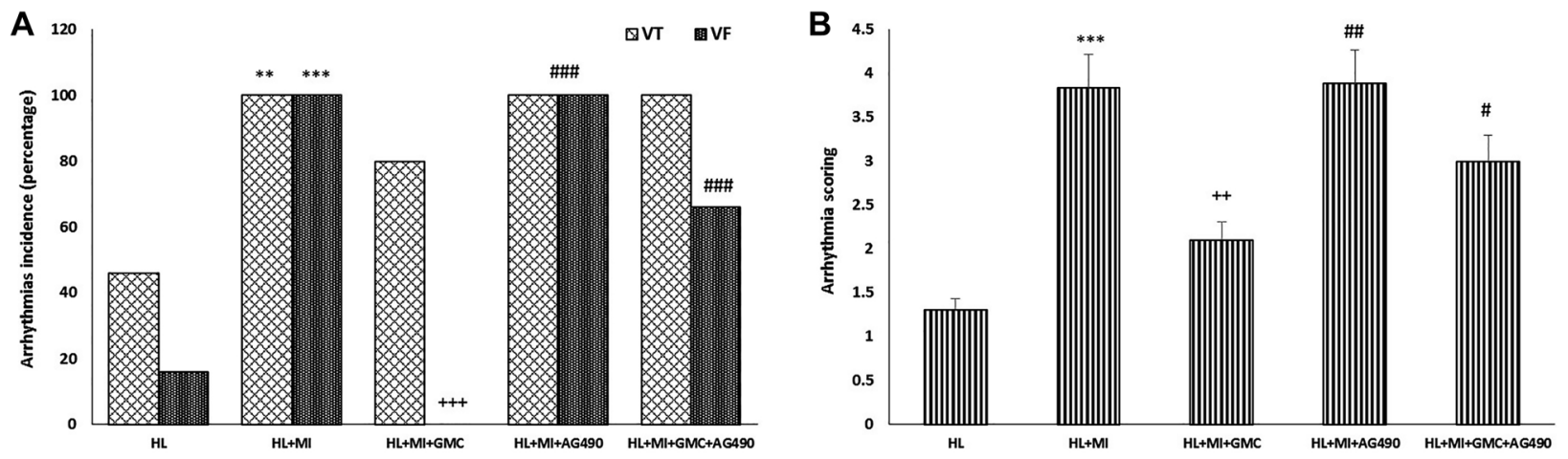

Figure 3: The effects of GMC administration on the incidence of VT and VF (A) and severity (B) of reperfusion-induced myocardial arrhythmias and their reversal by $A G 490$ in hyperlipidemic $(\mathrm{HL})$ rats.

Mean \pm SD ( $n=7$ in each group). MI, myocardial ischemia reperfusion injury; GMC, Ginsenoside-Mc1; AG490, JAK2 inhibitor; VT, ventricular tachycardia; and VF, ventricular fibrillation. ${ }^{\star \star} p<0.01,{ }^{\star \star \star} p<0.001$ vs. HL group; ${ }^{+++} p<0.001$ vs. HL + MI group; ${ }^{\#} p<0.05,{ }^{\# \#} p<0.01,{ }^{\# \# \#} p<0.001$ vs. $\mathrm{HL}+\mathrm{MI}+\mathrm{GMC}$ group.

HL rats, the amount of MDA increased significantly $(\mathrm{p}<0.001)$, and the level of SOD decreased $(\mathrm{p}<0.05)$ (Figure $5 \mathrm{~A}$ and $\mathrm{B}$ ). Despite this, GMC administration significantly prevented the effect of IR on changes in these two factors; it decreased MDA ( $\mathrm{p}<0.01)$ and increased SOD $(p<0.001)$ when compared to the HL + MI group. However, inhibition of the JAK2 pathway significantly neutralized GMC effects on oxidative stress markers following IR injury in the heart of HL rats ( $<<0.01)$ (Figure 5).

\section{GMC reduced IR-induced myocardial endoplasmic reticulum stress, whereas these effects were reversed by AG490 co-treatment}

Following IR injury, phosphorylation levels of PERK and eIF-2 $\alpha$ and protein expression of CHOP increased significantly compared to the HL group (Figure $6 \mathrm{~A}-\mathrm{C}$, respectively). This finding indicates an increase in ER stress following myocardial IR injury in hyperlipidemic rats. Pretreatment of HL rats with GMC prevented the upregulation of the expression of these ER stress proteins following IR insult $(p<0.01$, and $\mathrm{p}<0.001)$. Also, administration of AG490 as the inhibitor of the JAK2/STAT3 pathway was able to increase again the level of phosphorylation and expression of these proteins in comparison with the $\mathrm{HL}+\mathrm{MI}+\mathrm{GM}$ group $(\mathrm{p}<0.05)$ and neutralized the effect of GMC on ER stress in HL rats (Figure 5).

\section{GMC upregulated IR-induced reduction in myocardial JAK2/STAT3 expression, whereas these effects were reversed by AG490 co-treatment}

Phosphorylation of both JAK2 and STAT3 proteins was significantly reduced following induction of IR injury in 


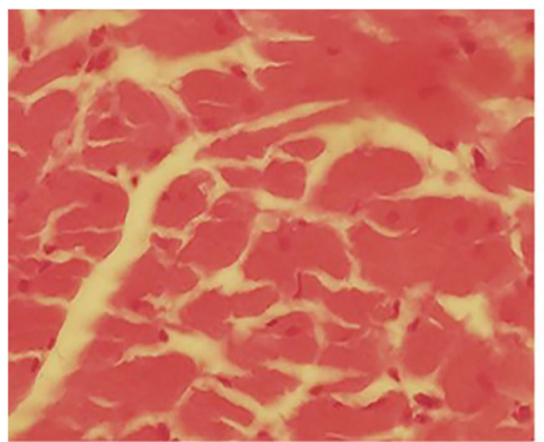

HL

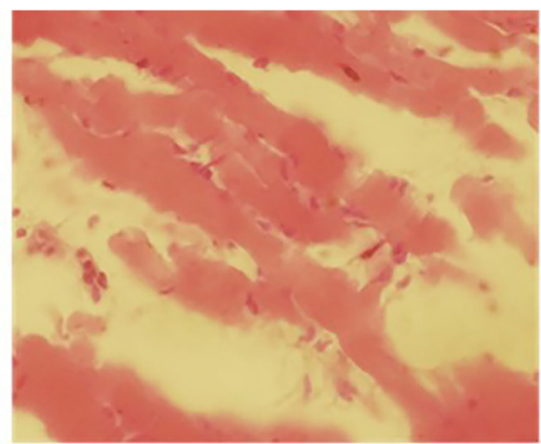

$\mathrm{HL}+\mathrm{MI}$

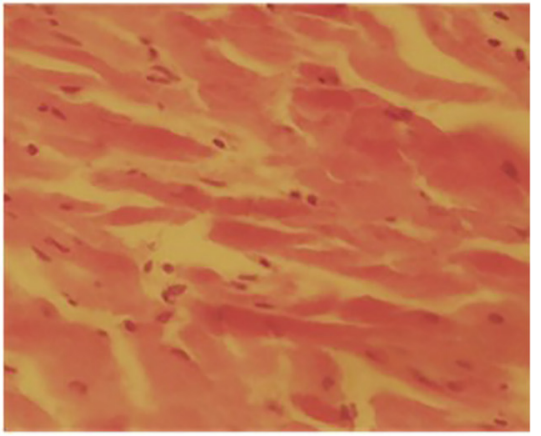

$\mathrm{HL}+\mathrm{MI}+\mathrm{GMC}$

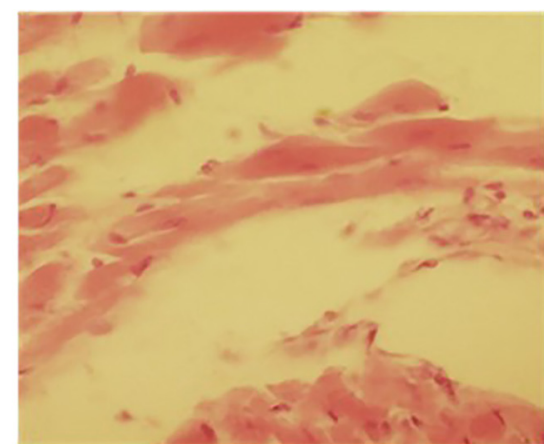

$\mathrm{HL}+\mathrm{MI}+\mathrm{AG} 490$

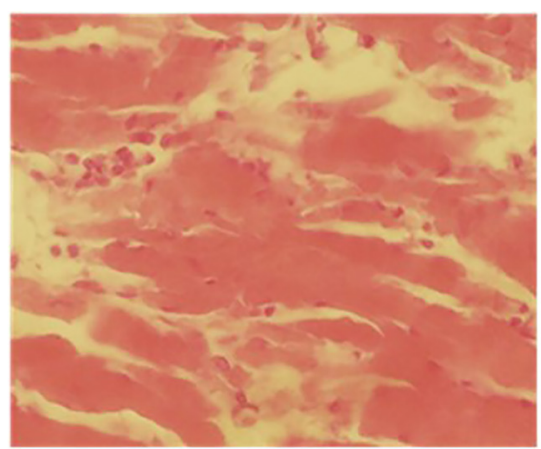

$\mathrm{HL}+\mathrm{MI}+\mathrm{GMC}+\mathrm{AG} 490$

Figure 4: The effects of GMC administration on myocardial histological changes induced by ischemia-reperfusion injury and their reversal by AG490 in hyperlipidemic (HL) rats.

40× magnification. MI, myocardial ischemia reperfusion injury; GMC, Ginsenoside-Mc1; AG490, JAK2 inhibitor.
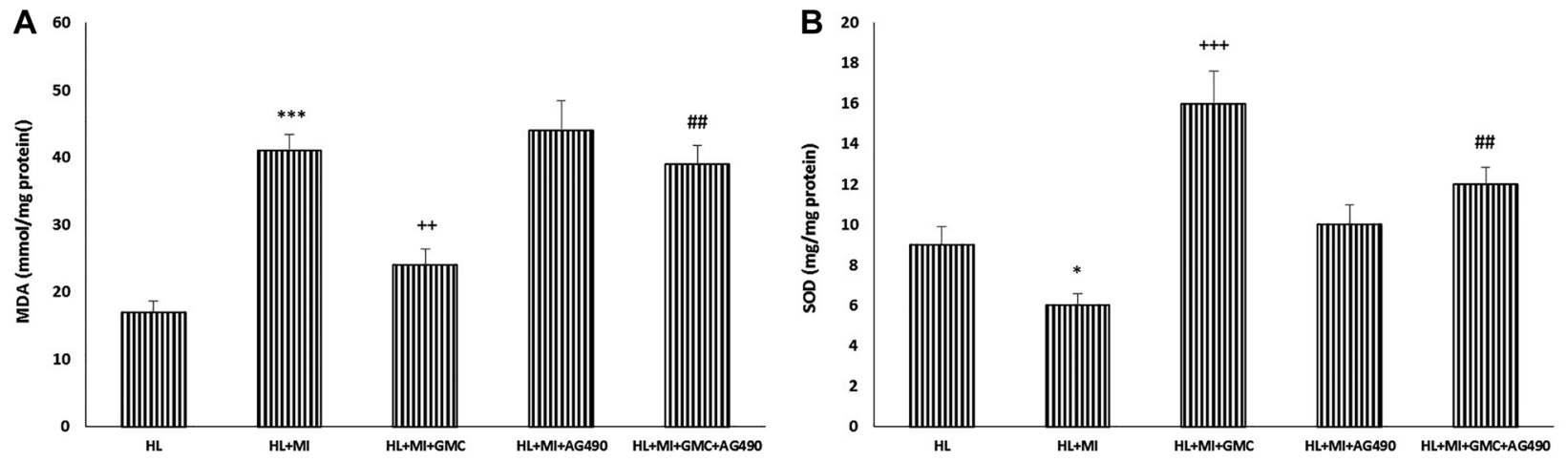

Figure 5: The effects of GMC administration on the levels of MDA (A) and SOD (B) following myocardial ischemia-reperfusion injury and their reversal by $A G 490$ in hyperlipidemic $(H L)$ rats.

Mean \pm SD ( $n=7$ in each group). MI, myocardial ischemia reperfusion injury; GMC, Ginsenoside-Mc1; AG490, JAK2 inhibitor. * $p<0.05$, ${ }^{\star \star \star} \mathrm{p}<0.001$ vs. HL group; ${ }^{++} p<0.01,{ }^{+++} p<0.001$ vs. $\mathrm{HL}+\mathrm{Ml}$ group; ${ }^{\# \#} \mathrm{p}<0.01$ vs. $\mathrm{HL}+\mathrm{MI}+\mathrm{GMC}$ group.

hyperlipidemic rats as compared to the untreated $\mathrm{HL}$ group (Figure 7A and B). Treatment of HL rats with GMC resulted in increased phosphorylation of JAK2/STAT2 proteins in the IR setting $(\mathrm{p}<0.001)$. Finally, although AG490 alone did not have a significant effect on JAK2 and STAT3 expression compared to the HL + MI group, its concomitant usage partially abolished the increasing effect of GMC on JAK2/STAT3 phosphorylation (Figure 7).

\section{Discussion}

In the present study, pretreatment of hyperlipidemic rats with GMC decreased the severity, duration, and number of reperfusion-induced myocardial arrhythmias following IR injury, as well as decreased myocardial oxidative stress indices and endoplasmic reticulum stress protein expression and increased the activity of the JAK2/STAT3 pathway. 

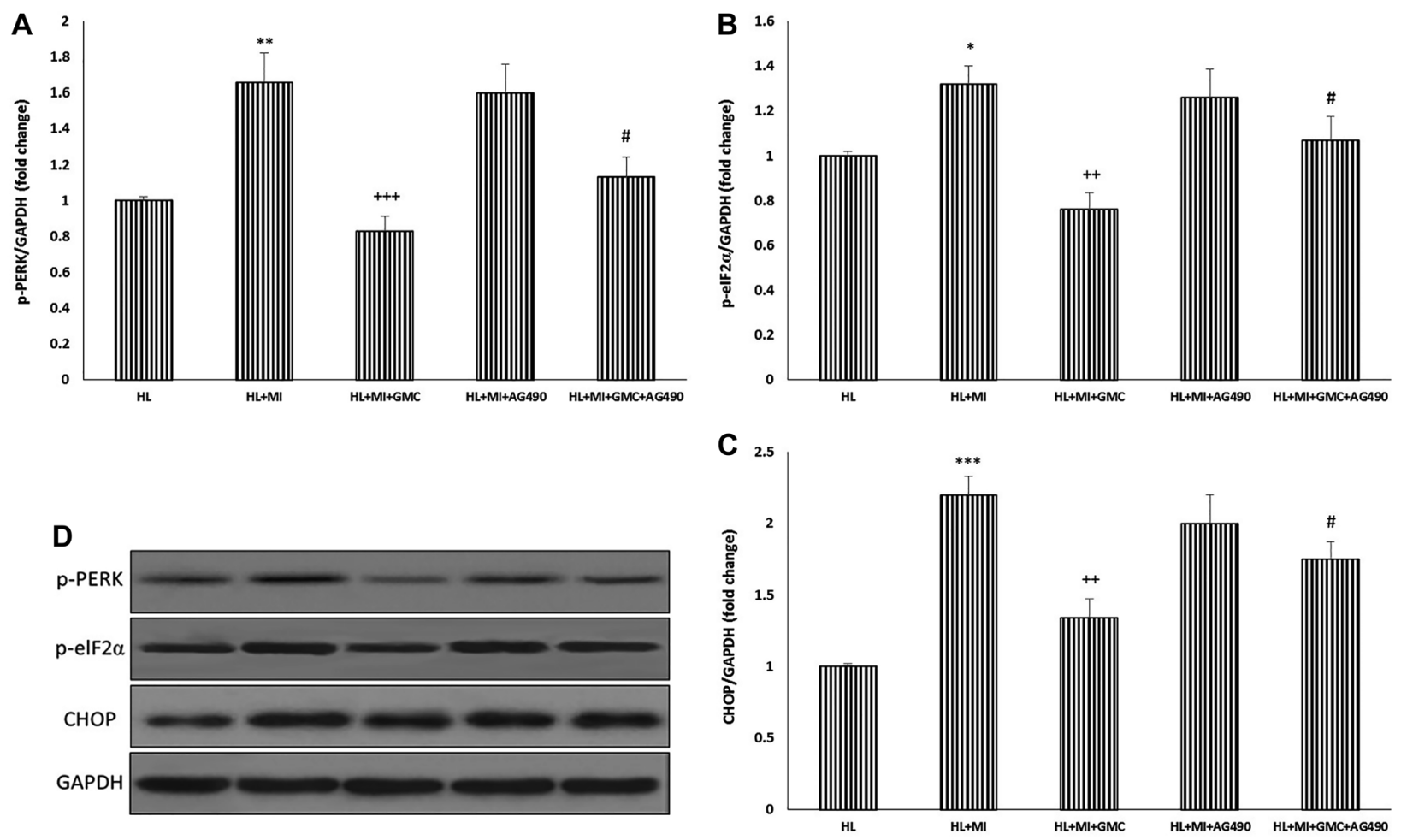

Figure 6: The effects of GMC administration on the expression of p-PERK (A), p-elF2 $\alpha(B)$, CHOP (C), and related representative bands (D) following myocardial ischemia-reperfusion injury and their reversal by AG490 in hyperlipidemic (HL) rats.

GAPDH was considered as an internal control of protein expression. Mean $\pm \mathrm{SD}$ ( $\mathrm{n}=7$ in each group). MI, myocardial ischemia reperfusion injury; GMC, Ginsenoside-Mc1; AG490, JAK2 inhibitor. ${ }^{\star} p<0.05,{ }^{\star \star} p<0.01,{ }^{\star \star \star} p<0.001 \mathrm{vs.} \mathrm{HL}$ group; ${ }^{++} p<0.01,{ }^{+++} p<0.001 \mathrm{vs}$. HL $+\mathrm{MI}$ group; \#p $<0.05$ vs. $\mathrm{HL}+\mathrm{MI}+\mathrm{GMC}$ group.

Besides, the results showed that by inhibiting JAK2 via concomitant administration of AG490, the cardioprotective effects of GMC in HL rats were abolished. Therefore, GMC demonstrated anti-arrhythmic properties and reduced endoplasmic reticulum stress and cellular oxidative stress by activating the JAK2/STAT3 pathway in the setting of myocardial IR injury in hyperlipidemic rats.

Fatal reperfusion arrhythmias are the hallmark of myocardial IR injury $[19,20]$. Sudden reperfusion to the ischemic tissue triggers a cascade of events such as oxidative stress, inflammatory cytokine production, imbalance in ionic homeostasis, and electrical activity which all finally may increase the occurrence of ventricular tachycardia and fibrillation leading to cardiac dysfunction and even death [3]. At the onset of reperfusion, marked inhomogeneity is seen in the electrical responses of the heart within the ischemic zone and areas at risk [21, 22]. Besides, hyperlipidemia also disturbs cardiac electrical activity directly, which may be related to myocardial ultrastructure changes, gradual accumulation of cardiac lipids, and consequent ER stress and oxidative stress [5]. This condition makes the heart vulnerable to IR injuries, leading to the induction of severe arrhythmias. In this line, our results showed that IR injury in hyperlipidemic rats considerably increased the number, duration, incidence, and scoring of ventricular arrhythmias, which are similar to the previous reports [9]. Arrhythmias usually occur in the early minutes of reperfusion, due to a variety of reasons, including the production of oxidative stress and the disruption of ionic and electrolyte balance in cardiomyocytes and dynamic organelles such as the mitochondria and ER. Here, the markers of ER and oxidative stress were greater and the activity of the JAK2/STAT3 pathway was lower in hyperlipidemic rats subjected to IR injury.

Accumulating evidence indicates that excessive ER stress may contribute to the occurrence of reperfusioninduced myocardial arrhythmias [10, 11]. PERK/eIF2 $\alpha /$ ATF 4/CHOP signaling pathway serves an essential role in ER stress and strongly causes lipid peroxidation, oxidative stress, and apoptotic cell death during IR injury, thereby reducing the ischemic tolerance of diabetic and hyperlipidemic hearts [12, 23]. When activated, PERK promotes phosphorylation of eIF2 $\alpha$ and subsequent translation of ATF4 and activation of CHOP. Overexpression of CHOP, in turn, activates downstream targets of ER stress, resulting in 

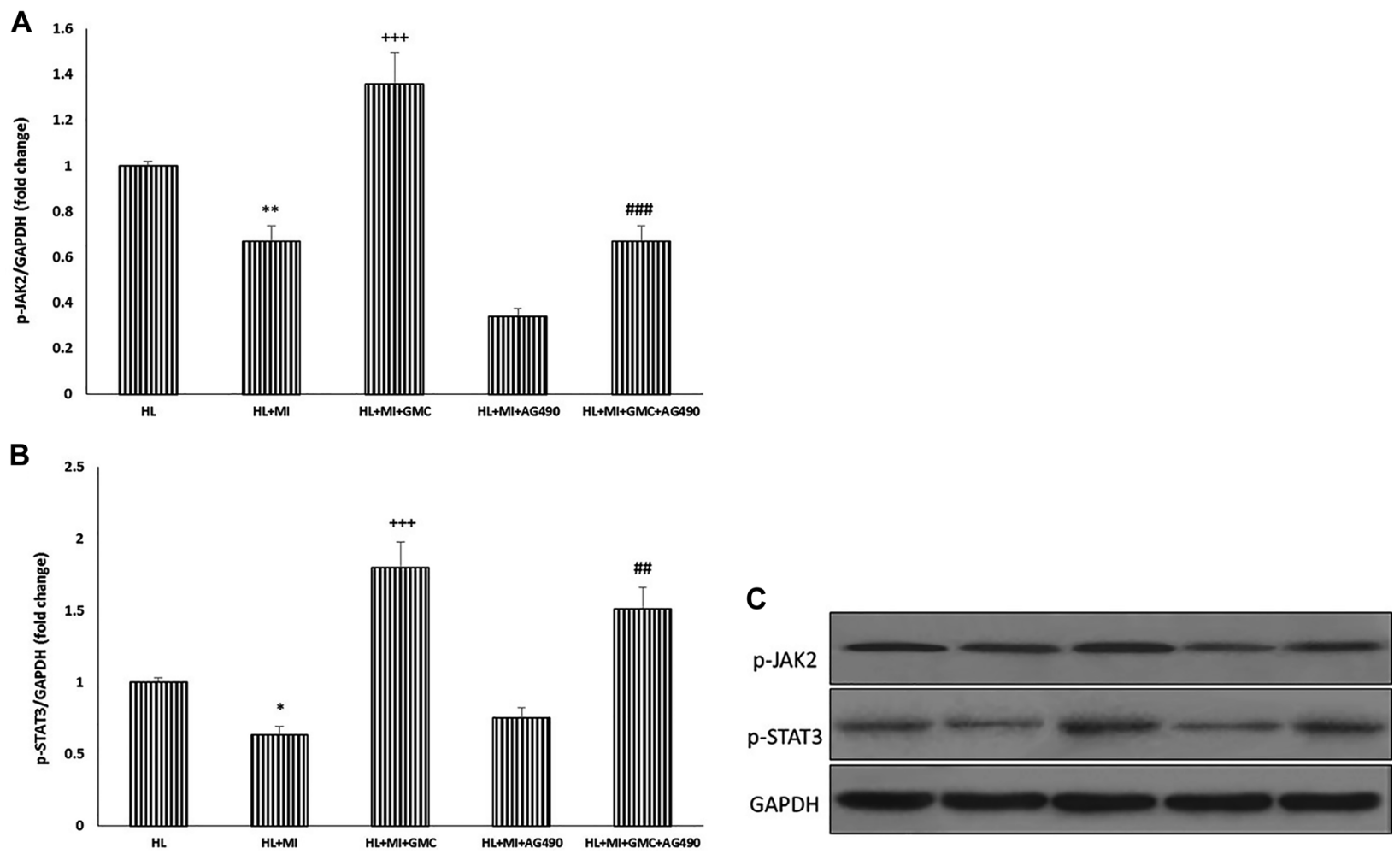

Figure 7: The effects of GMC administration on the expression of p-JAK (A), p-STAT3 (B), and related representative bands (C) following myocardial ischemia-reperfusion injury and their reversal by AG490 in hyperlipidemic $(\mathrm{HL})$ rats.

GAPDH was considered as an internal control of protein expression. Mean $\pm \mathrm{SD}$ ( $n=7$ in each group). MI: myocardial ischemia reperfusion

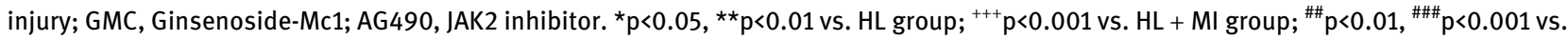
$\mathrm{HL}+\mathrm{MI}+\mathrm{GMC}$ group.

oxidative stress, apoptosis, and mitochondrial dysfunction $[18,24]$. Activation of unfolded protein response following ER stress may downregulate selective types of voltage gated sodium or potassium channels. This events increase the duration of ventricular action potential and reduce the rate of changes of action potential, contributing to electrical remodeling and ventricular arrhythmias [11, 25]. Pretreatment of hyperlipidemic rats with GMC (as a new de-glycosylated ginsenoside) significantly reduced the phosphorylation and activation of PERK/eIF2 $\alpha / \mathrm{CHOP}$ pathway in IR hearts. GMC also reversed IR-induced changes in the levels of lipid peroxidation marker MDA and exogenous antioxidative enzyme SOD. These effects of GMC were associated with the significant reduction in the counts, timing, incidence, and severity of ventricular arrhythmias including PVC, VT, and VF. In accordance with our findings, it has been recently reported that GMC attenuated oxidative stress and apoptosis in H9c2 cells cardiomyocytes through an AMPK dependent mechanism [14]. In addition, GMC reduced apoptosis and steatosis in the liver of high-fat diet-fed obese mice and prevented insulin resistance mostly by amelioration of the ER stress [16]. These findings demonstrated that the attenuation of ER stress and oxidative stress following IR injury by GMC pretreatment in hyperlipidemic rats are more likely involved in its antiarrhythmogenic potential.

Another feature of the cardioprotective effects of GMC in this study was the over-activation of the JAK2/STAT3 pathway along with suppression of IR-induced ER stress following GMC administration. More importantly, blockade of this pathway by AG490, as a specific inhibitor of JAK2, abolished the beneficial impacts of GMC on myocardial arrhythmias as well as oxidative and ER stress. It also impeded the positive impacts of GMC on IR-induced histopathological alterations. Therefore, these cardioprotective properties of GMC in hyperlipidemic rats are JAK2/STAT3 pathway-dependent. JAK2/STAT3 signaling is an important element of the SAFE pro-survival kinase signaling pathway in protecting the hearts against reperfusion injury and serves as a critical axis to mediate cardioprotective influences of ischemic and pharmacological conditionings $[6,8]$. In this scenario, JAK2/STAT3 pathway offers antioxidative and antiapoptotic effects to the hearts subjected to IR injury. Also, as a multifaceted transcription factor, STAT3 enhances 
the expression of genes that are involved in antioxidative, anti-inflammatory, proangiogenic, and antifibrotic processes [7]. Interestingly, more evidence indicates that activation of this pathway may attenuate ER stress both in in-vivo and in-vitro settings, and inhibition of the JAK2/STAT3 signaling pathway may reduce the clinical cardiovascular outcomes of atherosclerosis and hyperlipidemia [13, 26, 27]. Increased expression and activity of JAK2/STAT3 pathway by GMC led to antiarrhythmic effects in the early reperfusion by affecting the abovementioned arrhythmogenic elements. Accordingly, in the present study, AG490 administration neutralized the effect of GMC on the phosphorylation of PERK/eIF2 $\alpha$ and expression of CHOP. Also, GMC-induced JAK2 activation may improve cardiac function through correcting the histopathological changes following reperfusion phase. These findings postulate the immediate and gradual protective effects of GMC in IR injury which are JAK2/STAT3-dependent. It should be noted that although the use of AG490 alone did not have the significant impacts on the histopathological changes and biochemical parameters, it was able to considerably eliminate the effects of GMC to some extent. However, this inhibitory effect was not complete and the results suggest that there may be other parallel ways and mediators in the GMC's efficacy pathway that need further study. Also, to fully understand the mechanisms of GMC effects here, it is necessary to show how GMC upregulates JAK2/STAT3 pathway activity.

In conclusion, the findings of this study revealed that administration of GMC to the hyperlipidemic rats exerted substantial antiarrhythmogenic and histological benefits following myocardial IR injury. JAK2/STAT3 pathwaydependent attenuation of ER stress and oxidative stress mediated the cardioprotective effects of GMC. These findings may provide new mechanistic insight into the GMC effects in the presence of comorbidities and propose the possible implication of GMC in the management of reperfusion-induced arrhythmias.

Research funding: This study was supported by the Scientific Research Projects Commission of the First Clinical Medical College of Shanxi Medical University (project No. 2020084).

Author contribution: All authors contributed to the study design and conception, experiment conduction, data collection, and analysis. YZ wrote the first draft of the manuscript and all authors critically revised the manuscript. All authors read and approved the final manuscript.

Competing interests: Authors state no conflict of interest. Informed consent: Not applicable.
Ethical approval: All animal experiments and interventions in this study were performed in accordance with international ethical standards based on the approval of the University Ethics Committee (Ethics number: SDFRE2019023Dx).

\section{References}

1. Nowbar AN, Gitto M, Howard JP, Francis DP, Al-Lamee R. Mortality from ischemic heart disease. Circ Cardiovasc Qual Outcomes 2019;12:e005375.

2. Song R, Ren J, Sun J, Li M. Melatonin postconditioning combined with sitagliptin exerts full cardioprotection in diabetic hearts of aged rats through an AMPK-dependent mechanism. Arch Biol Sci 2021;73:83-92.

3. Kalogeris T, Baines CP, Krenz M, Korthuis RJ. Cell biology of ischemia/reperfusion injury. Int Rev Cell Mol Biol 2012;298: 229-317.

4. Sanada S, Komuro I, Kitakaze M. Pathophysiology of myocardial reperfusion injury: preconditioning, postconditioning, and translational aspects of protective measures. Am J Physiol Heart Circ Physiol 2011;301:H1723-41.

5. Andreadou I, Schulz R, Badimon L, Adameová A, Kleinbongard P, Lecour S, et al. Hyperlipidaemia and cardioprotection: animal models for translational studies. Br J Pharmacol 2020;177: 5287-311.

6. Sun XJ, Mao JR. Role of Janus kinase $2 /$ signal transducer and activator of transcription 3 signaling pathway in cardioprotection of exercise preconditioning. Eur Rev Med Pharmacol Sci 2018;22: 4975-86.

7. Harhous Z, Booz GW, Ovize M, Bidaux G, Kurdi M. An update on the multifaceted roles of STAT3 in the heart. Front Cardiovasc Med 2019;6:150

8. Kalakech H, Hibert P, Prunier-Mirebeau D, Tamareille S, Letournel F, Macchi L, et al. RISK and SAFE signaling pathway involvement in apolipoprotein A-I-induced cardioprotection. PLoS One 2014; 9: e107950.

9. Yao YS, Li TD, Zeng ZH. Mechanisms underlying direct actions of hyperlipidemia on myocardium: an updated review. Lipids Health Dis 2020;19:23.

10. Ruan Y, Zeng J, Jin Q, Chu M, Ji K, Wang Z, et al. Endoplasmic reticulum stress serves an important role in cardiac ischemia/ reperfusion injury (Review). Exp Ther Med 2020;20:268.

11. Liu M, Dudley SC Jr. The role of the unfolded protein response in arrhythmias. Channels 2018;12:335-45.

12. Sozen E, Ozer NK. Impact of high cholesterol and endoplasmic reticulum stress on metabolic diseases: an updated mini-review. Redox Biol 2017;12:456-61.

13. Li Y, Zhu W, Tao J, Xin P, Liu M, Li J, et al. Fasudil protects the heart against ischemia-reperfusion injury by attenuating endoplasmic reticulum stress and modulating SERCA activity: the differential role for PI3K/Akt and JAK2/STAT3 signaling pathways. PLoS One 2012;7:e48115.

14. Hong SH, Hwang HJ, Kim JW, Kim JA, Lee YB, Roh E, et al. Ginsenoside compound-Mc1 attenuates oxidative stress and apoptosis in cardiomyocytes through an AMP-activated protein kinase-dependent mechanism. J Ginseng Res 2020;44: 664-71. 
15. Lianqun J, Xing J, Yixin M, Si C, Xiaoming L, Nan S, et al. Comprehensive multiomics analysis of the effect of ginsenoside Rb1 on hyperlipidemia. Aging 2021;13:9732-47.

16. Roh E, Hwang HJ, Kim JW, Hong SH, Kim JA, Lee YB, et al. Ginsenoside Mc1 improves liver steatosis and insulin resistance by attenuating ER stress. J Ethnopharmacol 2020; 259:112927.

17. Karam I, Ma N, Yang Y-J, Li J-Y. Induce hyperlipidemia in rats using high fat diet investigating blood lipid and histopathology. J Hematol Blood Disord 2018;4:104.

18. Zhao GL, Yu LM, Gao WL, Duan WX, Jiang B, Liu XD, et al. Berberine protects rat heart from ischemia/reperfusion injury via activating JAK2/STAT3 signaling and attenuating endoplasmic reticulum stress. Acta Pharmacol Sin 2016;37:354-67.

19. Wit AL, Janse MJ. Reperfusion arrhythmias and sudden cardiac death: a century of progress toward an understanding of the mechanisms. Circ Res 2001;89:741-3.

20. van der Weg K, Majidi M, Haeck JD, Tijssen JG, Green CL, Koch KT, et al. Ventricular arrhythmia burst is an independent indicator of larger infarct size even in optimal reperfusion in STEMI. J Electrocardiol 2016;49:345-52.

21. Ng FS, Holzem KM, Koppel AC, Janks D, Gordon F, Wit AL, et al. Adverse remodeling of the electrophysiological response to ischemia-reperfusion in human heart failure is associated with remodeling of metabolic gene expression. Circ Arrhythm Electrophysiol 2014;7:875-82.

22. Sedova KA, Bernikova OG, Cuprova JI, Ivanova AD, Kutaeva GA, Pliss MG, et al. Association between antiarrhythmic, electrophysiological, and antioxidative effects of melatonin in ischemia/reperfusion. Int J Mol Sci 2019;20:6331.

23. Kadowaki $\mathrm{H}$, Nishitoh $\mathrm{H}$. Signaling pathways from the endoplasmic reticulum and their roles in disease. Genes 2013;4: 306-33.

24. Shang L, Dong P, Du L, Yang X, Wang H, Li S. SERP1 prevents hypoxia-reoxygenation-induced $\mathrm{H} 9 \mathrm{c} 2$ apoptosis through activating JAK2/STAT3 pathway-dependent attenuation of endoplasmic reticulum stress. Biochem Biophys Res Commun 2019;508:256-62.

25. Liu M, Dudley SC Jr. Role for the unfolded protein response in heart disease and cardiac arrhythmias. Int J Mol Sci 2015;17:52.

26. Wang $M$, Meng XB, Yu YL, Sun GB, Xu XD, Zhang XP, et al. Elatoside $C$ protects against hypoxia/reoxygenation-induced apoptosis in H9c2 cardiomyocytes through the reduction of endoplasmic reticulum stress partially depending on STAT3 activation. Apoptosis 2014;19:1727-35.

27. Yang X, Jia J, Yu Z, Duanmu Z, He H, Chen S, et al. Inhibition of JAK2/STAT3/SOCS3 signaling attenuates atherosclerosis in rabbit. BMC Cardiovasc Disord 2020;20:133. 